\title{
Utilización de un modelo de experimentación animal para la práctica de la Farmacoterapia en la docencia Veterinaria: Resultados preliminares
}

Sergio Villanueva-Saz ${ }^{a}$, María Jesús Muñoz Gonzalvob, José Javier Aramayona Alonso $^{c}$, Ana Rosa Abadía Valle ${ }^{\text {, }}$ Arantzazu Vitoria Moraiz

${ }^{a}$ Departamento de Farmacología y Fisiología. Facultad Veterinaria. Universidad de Zaragoza.

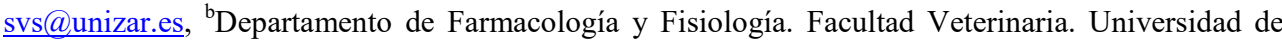
Zaragoza. mjmunoz@unizar.es, ${ }^{\mathrm{c} D}$ Departamento de Farmacología y Fisiología. Facultad Veterinaria. Universidad de Zaragoza. aramayon@unizar.es, ${ }^{\mathrm{d} D e p a r t a m e n t o ~ d e ~ F a r m a c o l o g i ́ a ~ y ~ F i s i o l o g i ́ a . ~}$ Facultad Veterinaria. Universidad de Zaragoza. arabad@unizar.es, e Departamento de Patología Animal. Facultad Veterinaria. Universidad de Zaragoza. avm@unizar.es.

\begin{abstract}
This work has been developed in Pharmacology and Pharmacotherapy subject of the third year of the Degree in Veterinary Medicine at the University of Zaragoza. Problem-Based Learning (PBL) with simulated cases has been used, providing a relatively large period of decision-making. This experience has been raised using a model of animal research for teaching of Pharmacology. Students, in groups, must put appropriate treatment in each of the phases of the implementation of the model (from the initial anesthesia to antibiotic treatment and final resolution process). In addition to applying the knowledge acquired in the subject, with teacher support in all cases, the student has had to make reasoned decisions about the problems that have arisen in real time. In assessing students it has taken into account the report of the actions motivated and rigor at work and teamwork. Finally, it has conducted a survey to students to know their degree of satisfaction with the experience gained.
\end{abstract}

Keywords: Veterinary, learning, Pharmacology, innovation, skills, solving problems, decision making.

\footnotetext{
Resumen

Este trabajo se ha desarrollado en la asignatura Farmacología y Farmacoterapia del tercer curso del Grado en Veterinaria de la Universidad de Zaragoza. En su docencia, durante mucho tiempo, se ha utilizado el Aprendizaje Basado en Problemas (ABP) con casos simulados y con un periodo de tiempo relativamente amplio para la toma de decisiones. En esta experiencia se ha planteado la utilización de un modelo de experimentación animal para que el estudiante, en grupos, instaurase el tratamiento adecuado en cada una de las fases de la implementación del modelo (desde la anestesia inicial al tratamiento antibiótico y la resolución final del proceso). Además de aplicar los conocimientos adquiridos en la asignatura, siempre con la tutorización del profesorado, el estudiante ha tenido que tomar decisiones razonadas sobre los problemas que han ido surgiendo en tiempo real. En la
} 
evaluación de los estudiantes de ha tenido en cuenta el informe motivado de las acciones realizadas, asi como el rigor en el trabajo y el trabajo en equipo. Finalmente, se ha realizado una encuesta al alumnado para conocer su grado de satisfacción con la experiencia realizada.

Palabras clave: Veterinaria, aprendizaje, farmacología, innovación, competencias, resolución de problemas, toma de decisiones.

\section{Introducción}

Entre los cambios producidos en la educación superior a raíz del proceso de convergencia en el Espacio Europeo de Educación Superior tiene "una especial importancia la adaptación de los programas antiguos por objetivos a programas por competencias y a la subordinación de los contenidos disciplinares a dichas competencias que, conceptualmente, son un "saber hacer complejo e integrador" (Fernández, 2006). Por otra parte, en 2004 el Grupo de Docencia y Formación de la Sociedad Española de Farmacología entre las recomendaciones sobre la docencia práctica de Farmacología en la formación de pre-grado (Baños et al., 2004) señalaba que "la formación práctica debería proporcionar al estudiante las habilidades necesarias para su futuro ejercicio profesional... ...conocimientos de las técnicas de estudio de los fármacos... ...deben utilizarse para fomentar el trabajo en equipo, la reflexión frente a los fenómenos biológicos y el autoaprendizaje. Además, es un excelente momento docente para adquirir actitudes deseables en el ejercicio profesional".

Son muchas las metodologías que ayudan a la formación en competencias de los futuros egresados (aprendizaje cooperativo, aprendizaje basado en problemas (ABP), aprendizaje por proyectos, método del caso, etc.). El equipo docente de este trabajo ha venido utilizando algunas de estas metodologías (Abadía et al., 2013), especialmente el ABP (Abadía et al., 2006), desde hace más de diez años, incluso en experiencias multidisciplinares (Abadía et al., 2012). En esta ocasión se ha planteado la utilización de un modelo de experimentación animal de fármacos como una práctica especial de Farmacología.

A pesar del desarrollo de métodos alternativos, los modelos de experimentación animal son necesarios para la realización de pruebas fundamentales en distintas disciplinas, entre ellas la Farmacología. No obstante, en este trabajo no se pretende introducir al estudiante en la investigación farmacológica, sino utilizar una experiencia de investigación habitual en la asignatura para que ellos apliquen los conocimientos que han adquirido sobre distintos fármacos. Por otra parte, la utilización de un modelo de experimentación animal puede contribuir a la transmisión al estudiante de la necesidad de un adecuado "cuidado y uso de los animales de laboratorio para alcanzar resultados válidos, confiables, reproducibles y comparables, así como a adoptar un actitud responsable desde el punto de vista ético y moral frente al uso de seres vivos" (Hernández, 2006)

(cc) EY-NC-ND 2016, Universitat Politècnica de València 
Finalmente, la utilización de una experiencia en la que el estudiante tiene que tomar decisiones, en tiempo real, para solucionar problemas que pueden ir surgiendo puede suponer un verdadero estímulo para que los propios estudiantes se impliquen en su propia formación.

\section{Objetivos}

El objetivo principal del presente trabajo ha sido que los estudiantes apliquen los conocimientos adquiridos en un modelo de experimentación que requiere la posible utilización de anestésicos, analgésicos, antibióticos, antipiréticos, antiinflamatorios, y otros fármacos en función de la evolución de cada experiencia.

Se pretende, además, que los estudiantes desarrollen otras competencias como observar, analizar, tomar decisiones, aplicar, reflexionar y trabajar en equipo.

Finalmente, se ha planteado analizar el grado de satisfacción del los estudiantes ante esta experiencia.

\section{Desarrollo de la innovación}

Esta experiencia se ha basado en la utilización de un modelo experimental tumoral en conejo para la investigación de fármacos antitumorales en las prácticas especiales de la asignatura Farmacología y Farmacoterapia del tercer curso del Grado en Veterinaria de la Universidad de Zaragoza (UZ). Cuenta con la aprobación de la Comisión Ética Asesora para la Experimentación Animal de la UZ.

Para el desarrollo de esta experiencia, todos los estudiantes matriculados en la asignatura (154) fueron divididos en grupos con un máximo de 7 alumnos por grupo. En cada sesión participaron varios profesores, si bien cada grupo tenía un tutor de referencia que le proporcionaba el apoyo necesario en el seguimiento postoperatorio.

La práctica tenía una duración total de 6 días y se desarrollaba de acuerdo con el siguiente calendario:

El primer día (día 1) el profesor responsable de la tutorización del grupo realizó una breve exposición de la práctica (objetivos, características del informe, procedimiento de trabajo, seguimiento diario de los animales). Después de la exposición, los estudiantes fueron llevados a la zona de pre-anestesia donde otro profesor explicó los conocimientos relacionados con manejo anestésico y cuidados preoperatorios necesarios antes de la cirugía.

Los estudiantes una vez realizadas las operaciones rutinarias previas a una intervención quirúrgica, realizaron la sedación y anestesia del animal combinando fármacos como medetomidina y ketamina. Posteriormente procedieron a la desinfección del campo quirúrgico. De forma paralela a estas maniobras, otros estudiantes del grupo se dedicaron a

(cc) EY-NC-ND 2016, Universitat Politècnica de València

Congreso In-Red (2016) 
la colocación del catéter disponiendo de una vía periférica para la colocación del gotero. A partir de este momento, el paciente estaba preparado para la cirugía de implantación del tumor.

Durante la cirugía experimental (Fig. 1), los profesores se encargaron de la intervención quirúgica, mientras que los estudiantes valoraban el estado vital del animal, asi como la necesidad o no de administrar algún tratamiento analgésico intraoperatorio. Una vez finalizada la cirugía, los estudiantes iniciaban la siguiente parte de la práctica o cuidados postoperatorios (eliminación del catéter, colocación de vendajes, control de la hipotermia y adaptación de la jaula a las necesidades postcirugía). Una de las premisas de esta práctica fue que los estudiantes del grupo serían los encargados de la calidad asistencial clínica del animal y de su cuidado.

Los estudiantes contaban en todo momento con la ayuda y el asesoramiento del profesor tutor del grupo. Para facilitar la comunicación entre el profesor y los estudiantes se introdujo la utilización de Whatsapp ${ }^{\circledR}$.

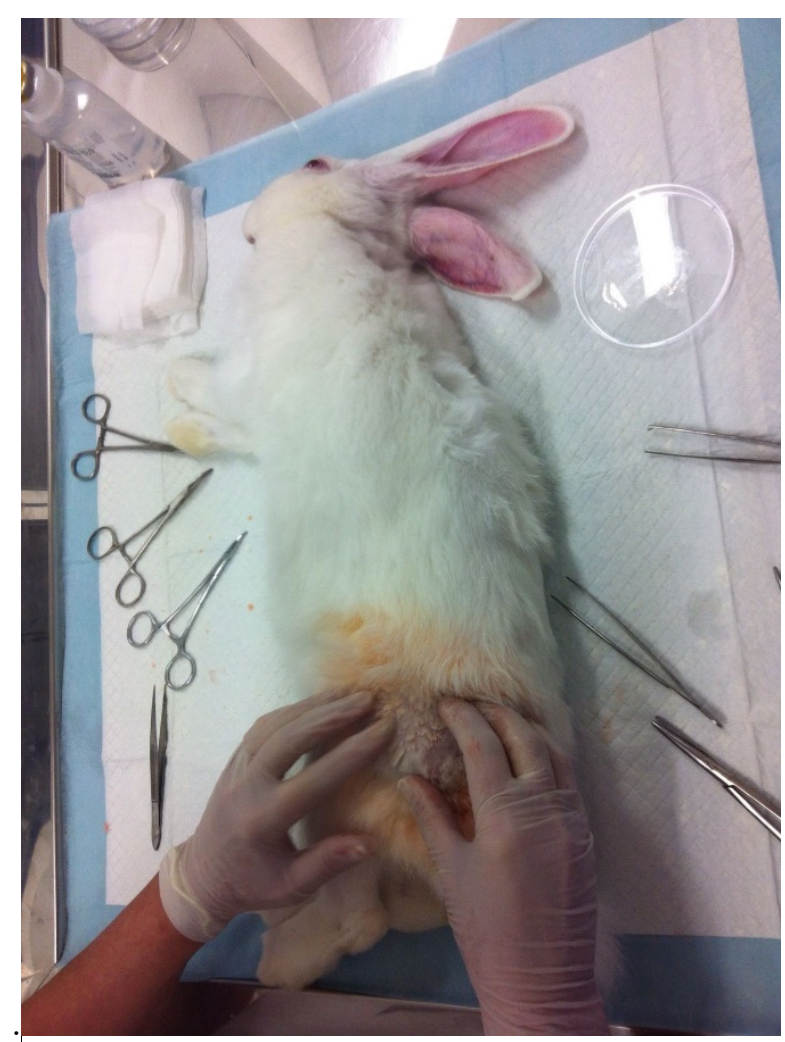

Fig. 1: Inicio de la cirugía

En los siguientes días (día 2, 3, 4 y 5), los estudiantes procedieron a la realización diaria de un examen físico (Fig. 2), junto a la toma de temperatura, limpieza de herida y colocación 
de nuevos vendajes, además de la administración parenteral de fármacos analgésicos (control del dolor) y antibioterapia (prevención de complicaciones infecciosas).

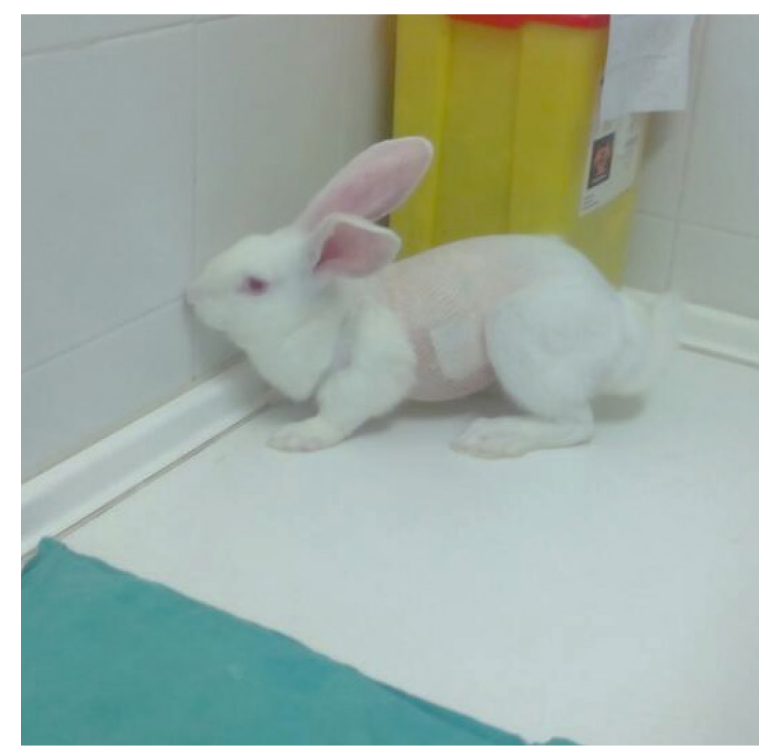

Fig. 2: Valoración del estado general del paciente

Finalmente el día 10, los estudiantes eliminaron las grapas quirúrigicas colocadas en el momento de la cirugía. Toda duda generada en relación con cualquier cuestión de la práctica fue resuelta por el profesor tutor.

Una vez finalizadas todas las partes prácticas de las diferentes sesiones, en el plazo máximo de cinco días (día 14), los estudiantes tenían que presentar un informe grupal, de formato libre. En él, debían recoger una explicación detallada de toda la práctica en su conjunto, dando especial importancia al manejo y utilización de los diferentes clases de fármacos empleadas, tanto en la preparación del paciente previo al procedimiento quirúrgico, como a los tratamientos famacológicos posteriores, incluyendo control del dolor. El informe también debía incluir una justificación de la selección de fármacos y aquellas situaciones clínicas que habían necesitado de la colaboración directa del profesor tutor para su resolución.

Una vez entregado el informe, se pidió a los estudiantes que contestasen a una encuesta constituida por varios grupos de preguntas. Un primer apartado inclúa preguntas relacionadas con la práctica realizada: novedad, dificultad, complejidad, satisfacción y eficiencia, entendida como la relación de la utilidad con el tiempo invertido. En este caso se utilizó una escala tipo "Likert" de 5 puntos: 1 (muy baja), 2 (baja), 3 (medio), 4 (alta) y 5 (muy alta).

Un segundo grupo de preguntas, puntuadas en una escala númerica de 0 (valor mínimo) y 10 (valor máximo), versaron acerca de la labor de los profesores participantes en la

(cc) EY-NC-ND 2016, Universitat Politècnica de València

Congreso In-Red (2016) 
práctica, el marco aplicativo de la práctica, la elaboración del informe y finalmente la nota global de la práctica en su conjunto.

Otro apartado, fue dedicado a valorar el grado de acuerdo de los estudiantes con afirmaciones planteadas en relación con la elaboración del informe que debían presentar al finalizar la práctica. Las cuestiones planteadas fueron: importancia de la guía del tutor en la realización del informe; dificultad para encontrar la información técnica necesaria para el informe; dificultad para sintetizar la información; capacidad para plasmar las ideas de forma escrita con orden, coherencia y claridad, así como valoración del formato del informe.. Se utilizó para ello una escala de 5 puntos: 1 (muy en desacuerdo), 2 (algo en desacuerdo), 3 (indiferente), 4 (algo de acuerdo) y 5 (muy de acuerdo).

También se quiso conocer la opinión de los estudiantes sobre si la utilización de formas alternativas de comunicación directa con el profesorado (Whatsapp ${ }^{\circledR}$ ) era algo positivo y les ayudaba a estar más seguros en la toma de decisiones terapéuticas a aplicar.

Una vez obtenidos los datos de las encuestas, se procedió a su análisis estadístico. Se realizó una distribución de frecuencias de las respuestas y se determinaron las medidas de tendencia central utilizando el programa SPSS.

Los resultados de las encuestas que se presentan en este trabajo son preliminares, y corresponden a las respuestas de los estudiantes matriculados en la asignatura "Farmacología y Farmacoterapia" en el curso académico 2015/2016 que han realizado la práctica hasta el 21 de mayo.

\section{Resultados}

A lo largo de esta experiencia todos los estudiantes han puesto en práctica más de una maniobra clínica que van a realizar cotidianamente en su futuro como profesionales veterinarios: manejo del animal, toma de temperatura corporal, administración de fármacos, curas de la herida quirúrgica y eliminación de grapas quirúrgicas. En relación con la asignatura en la que se ha desarrollado este trabajo, "Farmacología y Farmacoterapia", han tomado decisiones, en tiempo real, sobre la administración o no de antibióticos, analgésicos o antipiréticos en función del estado del paciente. La determinación de la dosis, intervalo de dosificación y vía de administración también ha sido realizada por los estudiantes, que han contado en todo momento con el apoyo y la supervisión de un profesor tutor.

La elaboración de un informe final incluyendo el razonamiento de las medidas terapéuticas tomadas en cada situación presentada ha contribuido a la profundización en el conocimiento de los grupos de fármacos utilizados.

La participación activa de los estudiantes en esta experiencia ha sido fundamental y el ambiente de trabajo creado en el grupo ha estado marcado por la motivación y la colaboración. 
En cuanto a la opinión de los estudiantes sobre los diferentes aspectos reflejados en la encuesta, hasta ahora se dispone de la información proporcionada por el $43.5 \%$ de los estudiantes matriculados (67). Como se ha indicado anteriormente, son datos preliminares.

Acerca de las características de la experiencia, de las respuestas de los estudiantes (Tabla 1) se deduce que la consideran novedosa (mediana 4), no muy difícil y de complejidad moderada (mediana 3). Se consideran satisfechos con la misma, y la consideran eficiente (mediana 4)

Tabla 1. Valoración de la experiencia. (Número de respuestas)

\begin{tabular}{|l|c|c|c|c|c|}
\hline \multirow{2}{*}{ Aspecto } & \multicolumn{5}{|c|}{ Escala de Likert } \\
\cline { 2 - 6 } & $\mathbf{1}$ & $\mathbf{2}$ & $\mathbf{3}$ & $\mathbf{4}$ & $\mathbf{5}$ \\
\hline Novedad & 0 & 0 & 0 & 31 & 32 \\
\hline Dificultad & 1 & 30 & 36 & 0 & 0 \\
\hline Complejidad & 0 & 16 & 46 & 5 & 0 \\
\hline Satisfacción & 1 & 2 & 9 & 29 & 26 \\
\hline Eficiencia & 0 & 3 & 15 & 40 & 9 \\
\hline
\end{tabular}

Los estudiantes han valorado globalmente la práctica con 8.4 puntos sobre 10 . La aplicación de los medicamentos antes, durante y después de la intervención quirúrgica realizada en esta experiencia les ha permitido desarrollar destrezas manuales, otorgándoles una puntuación de 8.7 sobre 10 en la encuesta realizada. La elaboración y la redacción del informe ha motivado una puntuación menor (7.6), si bien alcanza una calificación de notable. También han valorado muy positivamente la labor del profesorado en el desarrollo de la misma (8.8).

En cuanto a la redacción del informe (Tabla 2), los estudiantes consideran que la guía del tutor ha sido muy importante (mediana 5). No parece que hayan tenido dificultades para encontrar la información necesaria, ni para sintetizar y redactar el informe. Finalmente, ante la utilización de un formato libre proporcionado y la posibilidad de disponer de un formato prefijado, los estudiantes se muestran indiferentes (mediana 3).

(cc) EY-NC-ND 2016, Universitat Politècnica de València

Congreso In-Red (2016) 
Tabla 2. Valoración del informe. (número de respuestas)

\begin{tabular}{|l|c|c|c|c|c|}
\hline \multirow{2}{*}{ Aspecto } & \multicolumn{5}{c|}{ Escala de Likert } \\
\cline { 2 - 6 } & $\mathbf{1}$ & $\mathbf{2}$ & $\mathbf{3}$ & $\mathbf{4}$ & $\mathbf{5}$ \\
\hline $\begin{array}{l}\text { Importancia del papel del tutor como guía } \\
\text { en la elaboración del informe }\end{array}$ & 0 & 4 & 1 & 28 & 34 \\
\hline Dificultad para encontrar la información & 28 & 20 & 15 & 4 & 0 \\
\hline Dificultad de síntesis y redacción & 20 & 20 & 19 & 7 & 1 \\
\hline $\begin{array}{l}\text { Preferencia formato informe rígido y } \\
\text { prefijado }\end{array}$ & 10 & 10 & 30 & 12 & 5 \\
\hline
\end{tabular}

La supervisión necesaria del profesor tutor de las decisiones de los estudiantes sobre los tratamientos a aplicar llevó a la utilización de Whatsapp® como medio de comunicación entre ambos. Esta decisión ha sido considerada muy útil por la mayoría de los estudiantes participantes (99\%), reconociendo en un $85 \%$ de los casos, que la comunicación directa por esta vía les ha proporcionado más seguridad en la toma de decisiones. Los profesores consideran que la utilización de este tipo de herramienta de comunicación ha contribuido a favorecer la discusión clínica del caso.

\section{Conclusiones}

Se ha introducido un modelo experimental de desarrollo de un tumor en conejo en las actividades docentes de la asignatura Farmacología y Farmacoterapia, con el fin de que el que el estudiante ponga en práctica los conocimientos y destrezas adquiridos sobre analgésicos, anestésicos y antibióticos. Los estudiantes, trabajando en grupo, han tomado decisiones, siempre supervisados por el profesorado, sobre el fármaco, la dosis y la vía de administración más adecuada en cada caso. Han administrado el tratamiento y han efectuado su seguimiento, modificando los parámetros que han sido necesarios en función de los resultados obtenidos o de la evolución del animal. Todo ello son prácticas relacionadas con la Farmacología que tendrán que realizar en su futuro profesional. Además, la elaboración de un informe razonado sobre las actuaciones terapéuticas realizadas les ha permitido profundizar en el conocimiento de los grupos de fármacos utilizados.

La experiencia ha sido valorada positivamente por los estudiantes. La han considerado novedosa, no especialmente difícil y satisfactoria. Así mismo, muestran su reconocimento a labor del profesorado, y su importancia como guía en la elaboración del informe. 
Se ha favorecido el trabajo en grupo en un ambiente motivador y de colaboración entre los estudiantes, así como la comunicación profesor-estudiante, facilitada a través de WhatsApp para optimizar la rapidez en las respuestas.

\section{Agradecimientos}

A la Universidad de Zaragoza (UZ) por la concesión del proyecto de innovación docente PIIDUZ_15_168. A los estudiantes matriculados en la asignatura Farmacología y Farmacoterapia del Grado en Veterinaria de la UZ. Ana Rosa Abadía es coordinadora del Grupo de Investigación en Docencia e Innovación Universitaria (GIDIU) cofinanciado por el Gobierno de Aragón y el Fondo Social Europeo.

\section{Referencias}

Abadía, A.R., Aramayona, J.J., Muñoz, M.J. y Bregante, M.A. (2006) "Cómo aprenden Farmacología los estudiantes de Veterinaria de la Universidad de Zaragoza" En: Innovación docente, tecnologías de la información y la comunicación e investigación educativa en la Universidad de Zaragoza. Caminando hacia Europa Ed. Universidad de Zaragoza. 23 pp. Disponible en: $<$ http://www.unizar.es/eees/innovacion06/COMUNIC_PUBLI/BLOQUE_IV/CAP IV_28.pdf> [Consulta: 2 de abril de 2016]

Abadía, A.R., Loste, A., Bregante, M.A., Marca,M.C., Muñoz, M.J., Ortín, A. y Oliván, S. (2012) Compartiendo Aprendizaje Basado en Problemas. CIDUI 2012 - Llibre d'actes. La universidad: una institución de la sociedad. $22 \mathrm{pp}$. Disponible en: <http://www.cidui.org/revistacidui12/index.php/cidui/article/view/263 > [Consulta: 2 de abril de 2016]

ABADÍA, A.R, MUÑOZ, M.J, SIERRA, M, Y BUENO C. (2013) La técnica del puzzle en Farmacología Veterinaria. Univest 2013, 5 pp. Disponible en: <http://dugi-doc.udg.edu/handle/10256/8174> [Consulta: 2 de abril de 2016]

Baños, J.E., Bellido, I., Clos, M.V., Ivorra, D., Meana, J. y SÁnchez, S. (2004) "La docencia práctica de farmacología en la formación de pre-grado. Recomendaciones del Grupo de Docencia y Formación de la Sociedad Española de Farmacología” en Actualidad en Farmacología y Terapéutica, vol. 2, núm. 1, 70-71.

FERnÁNDEZ MARCH, A. (2006) "Metodologías activas para la formación de competencias" en Educatio siglo XXI, 24, 35-56.

HERNÁNDEZ, S. (2006) "El modelo animal en las investigaciones biomédicas” en Biomedicina, 2, 252256.

(cc)) EY-NC-ND 2016, Universitat Politècnica de València

Congreso In-Red (2016) 